\title{
Effects of age and physical activity on the autonomic control of heart rate in healthy men
}

R.C. Melo ${ }^{1}$, M.D.B. Santos ${ }^{1}$, E. Silva ${ }^{1,2}$, R.J. Quitério ${ }^{1}$, M.A. Moreno으. M.S. Reis ${ }^{2}$, I.A. Verzola ${ }^{1}$, L. Oliveira ${ }^{1}$, L.E.B. Martins ${ }^{3}$, L. Gallo-Junior ${ }^{4}$ and A.M. Catai ${ }^{1}$
Correspondence

A.M. Catai

Núcleo de Pesquisa em

Exercício Físico

Laboratório de Fisioterapia

Cardiovascular

Departamento de Fisioterapia, UFSCar

13565-905 São Carlos, SP

Brasil

Fax: +55-16-3351-2081

E-mail:mcatai@power.ufscar.br

Research supported by CAPES,

FAPESP, and CNPq

(No. 478799/2003-9).

Received May 10, 2004

Accepted April 14, 2005

\author{
${ }^{1}$ Núcleo de Pesquisa em Exercício Físico, Laboratório de Fisioterapia Cardiovascular, \\ Departamento de Fisioterapia, Universidade Federal de São Carlos, São Carlos, SP, \\ Brasil \\ ${ }^{2}$ Faculdade de Ciências da Saúde, Universidade Metodista de Piracicaba, \\ Piracicaba, SP, Brasil \\ ${ }^{3}$ Laboratório de Fisiologia do Exercício, Faculdade de Educação Física, \\ Universidade Estadual de Campinas, Campinas, SP, Brasil \\ ${ }^{4}$ Divisão de Cardiologia, Departamento de Clínica Médica, Hospital das Clínicas, \\ Faculdade de Medicina de Ribeirão Preto, Universidade de São Paulo, Ribeirão Preto, \\ SP, Brasil
}

\begin{abstract}
The effects of the aging process and an active life-style on the autonomic control of heart rate (HR) were investigated in nine young sedentary (YS, $23 \pm 2.4$ years), 16 young active (YA, $22 \pm 2.1$ years), 8 older sedentary (OS, $63 \pm 2.4$ years) and 8 older active (OA, $61 \pm 1.1$ years) healthy men. Electrocardiogram was continuously recorded for $15 \mathrm{~min}$ at rest and for $4 \mathrm{~min}$ in the deep breathing test, with a breath rate of 5 to 6 cycles/min in the supine position. Resting HR and RR intervals were analyzed by time (RMSSD index) and frequency domain methods. The power spectral components are reported in normalized units (nu) at low (LF) and high (HF) frequency, and as the $\mathrm{LF} / \mathrm{HF}$ ratio. The deep breathing test was analyzed by the respiratory sinus arrhythmia indices: expiration/inspiration ratio (E/I) and inspiration-expiration difference ( $\Delta \mathrm{IE})$. The active groups had lower HR and higher RMSSD index than the sedentary groups (life-style condition: sedentary $v s$ active, $\mathrm{P}<0.05)$. The older groups showed lower HFnu, higher LFnu and higher LF/HF ratio than the young groups (aging effect: young $v s$ older, $\mathrm{P}<0.05$ ). The OS group had a lower E/ I ratio (1.16) and $\triangle \mathrm{IE}(9.7 \mathrm{bpm})$ than the other groups studied (YS: 1.38, 22.4 bpm; YA: 1.40, 21.3 bpm; OA: 1.38, 18.5 bpm). The interaction between aging and life-style effects had a $\mathrm{P}<0.05$. These results suggest that aging reduces HR variability. However, regular physical activity positively affects vagal activity on the heart and consequently attenuates the effects of aging in the autonomic control of HR.
\end{abstract}

Key words

- Heart rate variability

- Respiratory sinus arrhythmia

- Autonomic nervous system

- Aging process

- Aerobic exercise 
Heart rate variability (HRV) has been used as a non-invasive tool to analyze the influence of the autonomic nervous system on the heart (1). The aging process reduces the parasympathetic activity on the heart and consequently, decreases the HRV indices (2,3-6). Moreover, increased predominance of the sympathetic activity over the parasympathetic balance is also observed at rest in older subjects (4). However, other findings suggest that the sympathovagal balance appears to be unchanged with aging due to decreases observed in both components (3). These observations are relevant since the reduction of HRV with aging can be related to higher cardiovascular morbidity and mortality rates $(7,8)$.

The methods utilized for HRV analysis include the time domain, which has been expressed as mean values and standard deviation of the RR interval duration, and the frequency domain, obtained from mathematical processing of the RR intervals in the electrocardiogram recording which can discriminate two main spectral components: high frequency (HF, ranging from 0.15 to $0.40 \mathrm{~Hz}$ ), and low frequency (LF, ranging from 0.04 to $0.15 \mathrm{~Hz}$ ), representative of parasympathetic and sympathetic control, respectively (1). However, the literature has reported that LF is influenced by both components (9).

Heart rate (HR) exhibits an oscillatory pattern in synchrony with the respiratory cycle, which is denominated respiratory sinus arrhythmia (RSA) (10-12). During inspiration, the HR increase is largely due to withdrawal of the parasympathetic activity on the sinus node. During expiration, the parasympathetic resumes activity and HR decreases (11). According to some investigators, RSA significantly decreases the sympathovagal balance, thus markedly increasing vagal modulation on the sinus node $(2,10,11)$.

The RSA is considered to be a representative sign of an intact cardiovascular sys- tem, showing the integrity of parasympathetic modulation on the sinus node $(2,10)$, notably more marked in the young than in older subjects $(2,10,11,13)$. It is currently believed that the aging process causes modifications in the cardiorespiratory system which may also alter other mechanisms indirectly involved in RSA genesis, i.e., the baroreflex and cardio-pulmonary stretch reflexes (11).

There are conflicting reports in the literature regarding the effects of aerobic training on HRV under resting conditions in both the time and frequency domains. While some studies have reported increases in HRV $(14,15)$, others have reported no change $(13,16,17)$. The intensity, duration and frequency of aerobic training appear to be directly related to HRV modifications. However, individual characteristics (genetic factors, fitness level, gender, age, chronic pathologies, etc.) are relevant to determine their magnitude (18). In general, regular aerobic physical activity improves functional capacity, produces resting bradycardia $(14,16$, $17)$ and may improve HRV $(14,15,19)$.

On the basis of these considerations, we hypothesized that long-term regular physical activity would attenuate the age-related decline in autonomic control of HR, which is relevant if we consider that higher cardiovascular morbidity and mortality rates are directly associated with reductions in HRV and functional capacity (7). Thus, the purpose of the present study was to investigate the effects of aging process and active lifestyle on the autonomic control of HR in healthy men.

Forty-one men volunteered to take part in this study. They were divided into the following four groups according to age and life-style (sedentary and active): young sedentary $(\mathrm{N}=9)$, young active $(\mathrm{N}=16)$, older sedentary $(\mathrm{N}=8)$, and older active $(\mathrm{N}=8)$. The young active group was involved in leisure activities (soccer, swimming, cycling, and running) twice a week, and the older 
active men had been participating in regular physical activities (running, cycling, swimming, and walking) over the last 15 years, at a frequency of 4 days per week (median value) with a duration of $60 \mathrm{~min}$ per session. All of them were in good health based on clinical and physical examination and laboratory tests that included a standard electrocardiogram (ECG), maximum exercise test conducted by a physician, chest X-ray, total blood count, urinalysis, and clinical biochemical screening tests (glucose, uric acid, total cholesterol and fractions, and triglycerides). All groups were composed of nonsmokers and none of the subjects studied were taking any type of medication. The subjects were informed about the experimental procedures and signed an informed consent form to participate in the study. The study was approved by the Ethics Committee of the Federal University of São Carlos.

All subjects were evaluated at the same time of day and at an experimental room temperature of $23^{\circ} \mathrm{C}$ and relative air humidity between 50 and $60 \%$. Before the day of the experiment, the subjects were taken to the experimental room for familiarization with the procedures and the equipment to be used. Each subject had been instructed to avoid caffeinated and alcoholic beverages and to avoid moderate or heavy exercise on the day before the application of the protocols. Before beginning the test on each experimental day, the subjects were interviewed and examined to confirm their state of good health, the occurrence of a normal night's sleep, and to confirm that the control conditions (HR and systemic blood pressure) were within the normal range.

First, the subjects were maintained at rest for $20 \mathrm{~min}$ for HR to return to control conditions. Then, 15 min of ECG data were obtained while subjects rested quietly, breathing spontaneously, in the supine position. Subsequently, the HR and RR interval were recorded during the deep breathing test, which was performed twice by each subject, for $4 \mathrm{~min}$, in the same position. During each test the subjects were instructed to perform deep forced inspirations and expirations, calmly and slowly, so that each cycle lasted $10 \mathrm{~s}$, i.e., $5 \mathrm{~s}$ for inspiration and $5 \mathrm{~s}$ for expiration. This procedure was based on the literature which advocates that cycles with 10- to 12-s duration, which correspond to a breath rate of 5 to 6 cycles/min are the most reliable to obtain maximal RSA (12). The subjects controlled their breath rate with a pointer clock and verbal feedback cues from the researchers. Real time feedback was given by the researchers based on observation of the visualized ECG signal on the computer screen which confirmed if the respiratory cycle had been performed in the correct way. At the end of the experiment, the subjects were instructed to breathe normally during the 1-min recovery. A resting period was allowed between tests to permit HR return to the control condition.

During all experiments the subjects were monitored at the CM5 lead to record the HR and RR intervals. The ECG and HR were obtained from a one-channel heart monitor (TC 500, ECAFIX, São Paulo, SP, Brazil) and processed using an analog-digital converter Lab. PC+ (National Instruments, Co., Austin, TX, USA), which represents an interface between the heart monitor and a Pentium III microcomputer. The signals were recorded in real time after analog to digital conversion and the RR interval (ms) was calculated on a beat-to-beat basis using specific software (20).

HRV was analyzed by the time and frequency domain methods. In the time domain, the RR intervals were analyzed by the RMSSD index, which corresponds to the square root of the mean sum of the squares of the difference between adjacent normal RR intervals in the record divided by the number of RR intervals within a given time minus one.

Before the frequency domain analysis, the highest stability section RR intervals, 
which included at least $5 \mathrm{~min}$, were selected for HRV analysis as a criterion required for the application of autoregressive spectral analysis. The power spectral components were then obtained at LF ( 0.04 to $0.15 \mathrm{~Hz})$ and $\mathrm{HF}$ ( 0.15 to $0.4 \mathrm{~Hz}$ ), in absolute units $\left(\mathrm{ms}^{2} / \mathrm{Hz}\right)$, and the normalized units were computed by dividing the absolute power of a given $\mathrm{LF}$ or $\mathrm{HF}$ component $\left(\mathrm{ms}^{2} / \mathrm{Hz}\right)$ by the total power, after subtracting from it the power of the component with a range frequency between 0 and $0.03 \mathrm{~Hz}$, i.e., very LF, and then multiplying this ratio by 100 . The $\mathrm{LF} / \mathrm{HF}$ ratio was also measured (1).

Frequency domain analysis was also applied to the RR intervals (ms) acquired during the deep breathing test and used to confirm if all subjects had maintained their breath rate at 5 to 6 cycles/min (i.e., peak of spectral density at a frequency ranging from 0.8 to $1.0 \mathrm{~Hz}$ ). Next, the RSA indices were calculated as follows:

Expiration/inspiration (E/I) ratio: the ratio was calculated by the mean value for the longest RR interval obtained during the expiration phase divided by the mean value for the shortest RR interval obtained during the inspiration phase of the deep breathing test $(2,10)$.

Inspiration-expiration difference $(\Delta \mathrm{IE})$ : the ratio was calculated by the difference between the mean value for the highest HR obtained during the inspiration phase and the mean value of the lowest HR obtained during the expiration phase of the deep breathing test $(2,10)$.

Data are reported as means \pm SD. The effect of aging (young vs older), life-style status (sedentary $v s$ active) and the interaction between aging and life-style were compared by two-way ANOVA for unpaired measurements. When appropriate, post hoc analyses were conducted using the Duncan test. The analysis was carried out using the Statistica for Windows software (computer program manual, StatSoft, Inc., 2000). The level of significance was set at $\mathrm{P}<0.05$.

Table 1 shows the anthropometric characteristics for the all groups studied. The older groups had a higher age and body mass index, and a lower height than the young groups (aging effect: young vs older, $\mathrm{P}<$ $0.05)$.

Table 2 shows the HR, RR intervals, time and frequency domain indices and the RSA indices. The active groups had higher RR intervals, RMSSD index and lower HR compared to the sedentary groups (life-style condition: sedentary $v s$ active, $\mathrm{P}<0.05)$. The frequency domain indices showed a significant aging effect (young $v s$ older), since the older groups had higher LF normalized units (LFnu), LF/HF ratio and lower HFnu than the young groups. Both RSA indices were influenced by interaction between aging (young $v s$ older) and life-style condition (sedentary $v s$ active). The older sedentary group had lower E/I ratio and $\Delta \mathrm{IE}$ than the two

Table 1. Comparison of the anthropometric data for all groups studied.

\begin{tabular}{|c|c|c|c|c|c|c|c|}
\hline & \multicolumn{2}{|c|}{ Young group } & \multicolumn{2}{|c|}{ Older group } & \multicolumn{3}{|c|}{$P$ values } \\
\hline & Sedentary $(\mathrm{N}=9)$ & Active $(N=16)$ & Sedentary $(\mathrm{N}=8)$ & Active $(\mathrm{N}=8)$ & A & $\mathrm{C}$ & 1 \\
\hline Age (years) & $23 \pm 2.4$ & $22 \pm 2.1$ & $63 \pm 2.4$ & $61 \pm 1.4$ & 0.00 & NS & NS \\
\hline Height $(\mathrm{cm})$ & $179 \pm 8.2$ & $182 \pm 4.6$ & $169 \pm 3.5$ & $170 \pm 6.1$ & 0.00 & NS & NS \\
\hline Weight (kg) & $77 \pm 8.9$ & $76 \pm 8.7$ & $72 \pm 6.5$ & $73 \pm 6.4$ & NS & NS & NS \\
\hline BMI $\left(\mathrm{kg} / \mathrm{m}^{2}\right)$ & $24 \pm 1.7$ & $23 \pm 1.9$ & $25 \pm 1.5$ & $25 \pm 1.2$ & 0.00 & NS & NS \\
\hline
\end{tabular}

Data are reported as means $\pm \mathrm{SD}$. BMI = body mass index; $\mathrm{A}=$ aging effect: young $v s$ older; $\mathrm{C}=$ life-style condition: sedentary vs active; I = interaction between aging effect and life-style condition; NS = nonsignificant. 
younger groups and older active group (interaction between aging effect and life-style condition, $\mathrm{P}<0.05)$.

The present study investigated differences in autonomic HR control as a function of aging and regular physical activity. Healthy older subjects compared to young subjects showed decreased parasympathetic activity and increased sympathetic activity at rest, estimated by HRV indices, and consequently, a higher sympathovagal balance. The active groups showed bradycardia at rest, which was associated with higher HRV. Furthermore, the older sedentary subjects had an attenuated HR response to the respiratory cycle (i.e., RSA indices) compared to the young and older active subjects. Thus, regular physical activity in the older subjects tended to reverse, in part, the age-related deficits in vagal activity on the heart.

The resting HR is modulated by both branches of the cardiac autonomic nervous system, with a predominance of parasympathetic influence (1). The vagal activity on the sinus node, estimated by multiple HRV indices, is decreased with age $(2-4,6,8)$. On the other hand, the literature reports that mean HR at rest does not increase with advancing age $(5,6)$, presumably due to the decrease in the intrinsic HR $(8,14)$ and the increase in sympathovagal balance (8). Our results support in part these previous observations, since no differences were found between young and older men with the same life-style conditions for mean HR at rest. However, in the present study we did not use combined parasympathetic and sympathetic pharmacological blockade and consequently cannot make any statement about intrinsic HR.

It has been shown that HRV declines with aging (2-4,6,8), a fact mainly (4), but not exclusively, due to a reduction in parasympathetic activity. On the other hand, the sympathetic activity also declines with advanced age, but at a slower rate (8). In the present study, the older subjects had lower vagal activity (i.e., low HFnu) which, associated with the higher LFnu, may have con-

Table 2. Comparison of the heart rate variability indices in time and frequency domain analysis during the resting supine condition, and respiratory sinus arrhythmia indices calculated by the deep breathing test, between all groups studied.

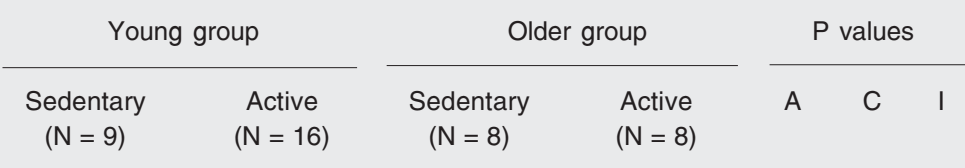

\begin{tabular}{|c|c|c|c|c|c|c|c|}
\hline \multicolumn{8}{|l|}{ Rest } \\
\hline \multicolumn{8}{|l|}{ Time domain } \\
\hline $\mathrm{HR}(\mathrm{bpm})$ & $70 \pm 9.3$ & $59 \pm 5.6$ & $68 \pm 6.7$ & $56 \pm 8.9$ & NS & 0.00 & NS \\
\hline RR interval (ms) & $874 \pm 123.8$ & $1034 \pm 100.3$ & $893 \pm 93.8$ & $1109 \pm 186.3$ & NS & 0.00 & NS \\
\hline RMSSD index (ms) & $34 \pm 14.6$ & $63 \pm 19.9$ & $27 \pm 9.2$ & $48 \pm 22.4$ & NS & 0.00 & NS \\
\hline \multicolumn{8}{|l|}{ Frequency domain } \\
\hline LFnu & $35 \pm 16.9$ & $27 \pm 12.2$ & $46 \pm 7.4$ & $43 \pm 9.9$ & 0.01 & NS & NS \\
\hline HFnu & $65 \pm 16.9$ & $72 \pm 12.2$ & $54 \pm 7.4$ & $56 \pm 9.9$ & 0.01 & NS & NS \\
\hline LF/HF & $0.65 \pm 0.6$ & $0.41 \pm 0.3$ & $0.87 \pm 0.2$ & $0.81 \pm 0.3$ & 0.01 & NS & NS \\
\hline \multicolumn{8}{|l|}{ Deep breathing test } \\
\hline$E / I$ & $1.38 \pm 0.11$ & $1.40 \pm 0.15$ & $1.16 \pm 0.04^{\star+}$ & $1.38 \pm 0.11$ & 0.00 & 0.00 & 0.01 \\
\hline$\Delta \mathrm{IE}(\mathrm{bpm})$ & $22 \pm 2.7$ & $21 \pm 7.0$ & $10 \pm 2.1^{*+}$ & $18 \pm 5.9$ & 0.00 & 0.04 & 0.01 \\
\hline
\end{tabular}

Data are reported as means $\pm \mathrm{SD}$. HR = heart rate; LFnu = low frequency in normalized units; HFnu = high frequency in normalized units; $\mathrm{LF} / \mathrm{HF}=$ low frequency/high frequency ratio; $\mathrm{E} / \mathrm{I}=$ expiration/inspiration ratio; $\Delta \mathrm{IE}=$ inspiration-expiration difference; $\mathrm{A}=$ aging effect: young vs older; $\mathrm{C}=$ life-style condition: sedentary vs active; I = interaction between aging effect and life-style condition; NS = nonsignificant.

${ }^{*} \mathrm{P}<0.05$ compared to young groups. ${ }^{+} \mathrm{P}<0.05$ compared to sedentary and active older groups. 
tributed to the greater sympathovagal balance observed. However, as the LF component is also influenced by the parasympathetic branch (9), we could not quantify the specific contribution of sympathetic efferent activity to the resting HRV.

Although the rest bradycardia observed after aerobic training has been extensively studied, the mechanisms involved in this alteration are unclear $(16,17)$. Leicht et al. (13) suggest that the increases in the HF component of the HRV are responsible for the reduced HR at rest. Other studies consider resting bradycardia to be much more related to changes in intrinsic HR than to modifications of efferent sympathovagal modulation $(16,17)$. The lower resting HR values observed in both active groups in the present study were also associated with high vagal activity, which could be represented by greater HRV indices. These findings can produce improvements in cardiovascular health, most likely due to the effects of regular physical activity. However, the nonpharmacological methods utilized in the present study do not permit us to make statements about the mechanism that induces bradycardia.

The literature also reports that regular physical activity enhances the HRV evaluated in the time domain for young $(14,18)$ and older subjects $(14,19)$. Our results agree with those of these studies, with the HRV analyzed by time domain for both active groups being higher than the HRV of agematched sedentary groups. Furthermore, the older active group showed similar values for the RMSSD index in comparison to the young active group. The long-term physical activity performed by the older active group probably contributed to these findings and must be taken into consideration.

On the other hand, when the HRV was analyzed in the frequency domain, no differences were found between the active and sedentary groups. In spite of the different results obtained in time and frequency do- main analysis, it is important to note that the RMSSD should be considered to be a marker of overall HRV (9) while a non-invasive contribution by each division of autonomic modulation to HRV is possible when it is represented in its frequency domain. However, the normalization tends to minimize the effect of the changes in total power on the values of the LF and HF components $(1,9)$. In addition, the use of normalized units or of the $\mathrm{LF} / \mathrm{HF}$ ratio does not take into account the fact that the LF component is not a pure indicator of sympathetic activity and that parasympathetic stimulation is also capable of producing variations of HR in the frequency range of the LF component (9). Thus, the higher vagal activity observed here in the older active group might have influenced the LF component that was responsible for the elevated LFnu and LF/HF ratio, explaining the lack of difference between sedentary and active older groups when the frequency component was expressed in normalized units or as the LF/HF ratio.

The RSA indices are utilized as a tool for analysis of vagal modulation of the sinus node $(2,10,17)$ and consequently to evaluate autonomic dysfunction $(2,10)$. Some investigators have reported that the $\mathrm{E} / \mathrm{I}$ ratio is reduced with the aging process $(2,10)$ due to decreases in the vagal response synchronized with the respiratory cycle. In the same way, the inspiration-expiration difference $(\Delta \mathrm{IE})$ is also a sensitive index of autonomic dysfunction (10) and may be directly correlated with vagal activity on the heart and indirectly correlated with aging. Moreover, the RSA is higher in adults who exercise routinely and attenuated in older subjects $(11,19)$, patient with coronary disease and diabetic patients (11). The present findings agree with the literature $(2,10)$, i.e., the E/I ratio and $\triangle \mathrm{IE}$ were lower in the older sedentary group than in the young sedentary group, confirming the influence of aging on these indices.

The aging process is considered to cause 
structural and neuronal modifications in the cardiorespiratory system, which could alter some mechanisms indirectly involved in RSA genesis (i.e, baroreflex, chemoreflex and cardio-pulmonary stretch reflex) (11). The higher $\mathrm{E} / \mathrm{I}$ ratio and $\Delta \mathrm{IE}$ observed in the older active group compared to the older sedentary group led us to believe that some of the mechanisms involved in the RSA as well as in vagal activity could be positively influenced by the physical activity in the older group. Thus, in the present study, the RSA indices showed the interaction between aging and regular physical activity, suggesting that an active life pattern is effective in attenuating the aging decline of HRV. These observations are relevant if we consider that a lower HRV is associated with an increased risk of cardiac events $(7,8)$.

The present study has some limitations. First, the breath rate at rest was not controlled and the tidal volume during the deep breathing test was not measured; thus, the relative and absolute stimulus imposed by controlled breathing could not be analyzed (17). Second, despite the self-reported habitual physical level utilized in present study, nothing can be inferred about frequency, duration and intensity of the aerobic activity performed by the young and older active groups. However, the long periods involved in a regular activity might have contributed to all the present findings.

The results of the present investigation suggest that the aging process causes a decrease in HRV. Moreover, regular physical activity has positive effects on the vagal activity on the heart and consequently attenuates the effects of aging on the autonomic control of HR when it is evaluated by HRV and by RSA indices. Therefore, further studies on the correlation between physical activity and aging process regarding the autonomic control of HR and the mechanisms of RSA are needed.

\section{Acknowledgments}

The authors are grateful to Prof. Carlos Alberto Ribeiro Diniz, Departamento de Estatística, Universidade Federal de São Carlos, for assistance with the statistical analysis.

\section{References}

1. Task Force of the European Society of Cardiology of the North American Society of Pacing Electrophysiology (1996). Heart rate variability standards of measurement, physiological interpretation, and clinical use. Circulation, 93: 1043-1065.

2. Gautschy B, Weidmann P \& Gnãdinger MP (1986). Autonomic function tests as related to age and gender in normal man. Klinische Wochenschrift, 64: 499-505.

3. Pagani M, Lombardi F, Guzzetti S et al. (1986). Power spectral analysis of heart rate and arterial pressure variabilities as a marker of sympatho-vagal interaction in man and conscious dog. Circulation Research, 59: 178-193.

4. Lipsitz LA, Mietus J, Moody GB \& Goldberger AL (1990). Spectral characteristics of heart rate variability before and during postural tilt. Relations to aging and risk of syncope. Circulation, 81: 1803-1810.

5. Lakatta EG \& Levy D (2003). Arterial and cardiac aging: major shareholders in cardiovascular disease enterprises. Part II: the aging heart in health: links to heart disease. Circulation, 107: 346-354.

6. Jensen-Urstad K, Storck N, Bouvier F, Ericson M, Lindblad LE \& Jensen-Urstad M (1997). Heart rate variability in healthy subjects is related to age and gender. Acta Physiologica Scandinavica, 160:
235-241.

7. Bigger JT, Fleiss JL, Steinman RC, Rolnitzky LM, Kleiger RE \& Rottman JN (1992). Frequency domain measures of heart period variability and mortality after myocardial infarction. Circulation, 85: 164-171.

8. Semrád B, Fiser B \& Honzíková N (1998). Ageing and cardiac autonomic status. In: Malik M (Editor), Clinical Guide to Cardiac Autonomic Tests. Kluwer Academic Publishers, Dordrecht, Boston, London, 285-300.

9. Hartikainen JEK, Tahvanainen KUO \& Kuusela TA (1998). Shortterm measurement of heart rate variability. In: Malik M (Editor), Clinical Guide to Cardiac Autonomic Tests. Kluwer Academic Publishers, Dordrecht, Boston, London, 149-176.

10. O'Brien IA, O'Hare P \& Corrall RJM (1986). Heart rate variability in healthy subjects: effect of age and the derivation of normal ranges for tests of autonomic function. British Heart Journal, 55: 348-354.

11. Yasuma $F$ \& Hayano $J$ (2004). Respiratory sinus arrhythmia. Why does the heartbeat synchronize with respiratory rhythm? Chest, 125: 683-690.

12. Hayano J, Mukai S, Sakakibara M, Okada A, Takata K \& Fujinami T 
(1994). Effects of respiratory interval on vagal modulation of heart rate. American Journal of Physiology, 267: H33-H40.

13. Leicht AS, Allen GD \& Hoey AJ (2003). Influence of age and moderate-intensity exercise training on heart rate variability in young and mature adults. Canadian Journal of Applied Physiology, 28: 446461.

14. Levy WC, Cerqueira MD, Harp GD, Johannessen K, Abrass IB, Schwartz RS \& Stratton JR (1998). Effect of endurance exercise training on heart rate variability at rest in healthy young older men. American Journal of Cardiology, 82: 1236-1241.

15. Stein PK, Ehasin AA, Domltovich PP, Kleiger RE \& Rottman JN (1999). Effect of exercise training on heart rate variability in healthy older adults. American Heart Journal, 138: 567-576.

16. Catai AM, Chacon-Mikahil MPT, Martinelli FS et al. (2002). Effects of aerobic exercise training on heart rate variability during wakefulness and sleep and cardiorespiratory responses of young and middle-aged healthy men. Brazilian Journal of Medical and Biological Research, 35: 741-752.

17. Scoot AS, Eberhard A, Ofir D, Benchetrit G, Dinh TP, Calabrese P, Lesiuk V \& Perrault H (2004). Enhanced cardiac vagal efferent activity does not explain training-induced bradycardia. Autonomic Neuroscience: Basic and Clinical, 112: 60-68.

18. Melanson EL (2000). Resting heart rate variability in men varying in habitual physical activity. Medicine and Science in Sports and Exercise, 32: 1894-1901.

19. De Meersman RE (1993). Heart rate variability and aerobic fitness. American Heart Journal, 125: 726-731.

20. Silva E, Catai AM, Trevelin LC, Guimaraes JO, Silva Junior LP, Silva LMP, Oliveira L, Milan LA, Martins LEB \& Gallo Junior L (1994). Design of a computerized system to evaluate the cardiac function during dynamic exercise. Physics in Medicine and Biology, 39a: 409 (Abstract). 